\title{
Risk of caries and oral health: preliminary study
}

\section{Marcello Gatti, Tatiana Giulia Rizzati, Francesca Scandurra}

Dipartimento di Scienze Odontostomatologiche, sezione di Microbiologia, Alma Mater Studiorum, Università degli Studi di Bologna

Key Words: risk of caries, S. mutans, Oral healt

Soggetti a rischio di carie e salute orale: studio preliminare

\section{SUMMARY}

Introduction. The incidence of injury cariosa remains high, despite the improvements achieved in the last years. Recent national epidemiological surveys, 4 years old children have healthy teeth in $80 \%$ of cases at 12 years the percentage is reduced to $50 \%$. In Italy, the almost total absence on the territory of "dental services to the Community", makes even more difficult to achieve a solution to the problem "caries." To address this problem, the Ministry of Labor, Health and Social Policy in October 2008 adopted the national guidelines in order to make suggestions to the various professionals (pediatricians, dentists, microbiologists, dental hygienists, etc.), making them interact to maintain and restore oral health. It was the first time that the Ministry of Health has addressed the problem by inserting the figure of the microbiologist in dentistry.

Aims. The present study aim was to identify subjects at risk of caries by clinical microbiological testing of saliva and the index DMFT/dmft (Decayed, Missing and Filling Permanent Teeth) in both adults and particularly children in order to take preventive measures early as reported in "National guidelines for the promotion of oral health and prevention of oral diseases in age of development".

The study began in June 2009 and will last one year with as goal to have, in 2010,90\% of children between 5 and 6 years caries free and 18 years with any lost tooth decay.

Materials and methods. Recruited 164 patients were divided into three age groups: I 24 adults aged between 20 and 40 years, 40 children which 21 till 5 years old and 19 till 12 years old.

Microbiological testing was aimed by finding CFU / $\mathrm{ml}$ of saliva of Streptococcus mutans and Lactobacillus spp., Slide CRT bacteria (Ivoclar). Clinically, intraoral examination was performed to evaluate the DMFT (Decayed teeth, Missing or Filling) calculated over 28 permanent teeth and the $\mathrm{dmft}$ (decayed teeth, missing or filling) calculated on 20 deciduous teeth in children, using the form 's WHO.

Results. $35.97 \%$ of the samples tested positive with a high bacterial count> $10^{5} \mathrm{CFU} / \mathrm{ml}$ of saliva concerning S.mutans and $31.70 \%$ were positive with a high bacterial count $>10^{5} \mathrm{CFU} / \mathrm{ml}$ of saliva concerning Lactobacillus spp. The DMFT of adults was equal to 2 .I, the one of children of 12 years was equal to 1.5 and the one of children of 4 years was equal to 1.01 .

Conclusions. The results confirmed the importance of checking regularly, at least 2 times a year, individuals who are charged with critical S.mutans and Lactobacillus spp. through microbiological testing of saliva and through DMFT / dmft indexes for early detection of individuals at risk of caries.

\section{INTRODUZIONE}

La carie dentaria è ancora oggi tra le patologie più diffuse nella popolazione generale $(31,22,36,12,24,27)$ e in età pediatrica $(28,4,6,5,3)$. Da recenti indagini epidemiologiche a carattere nazionale si evidenzia che il problema carie è ancora pressante nei bambini italiani: emerge una prevalenza di circa il $22 \%$ di patologia a 4 anni e di circa il $44 \%$ a 12 anni $(29,3)$. In Italia, la quasi totale assenza sul territorio di "Servizi Odontoiatrici di Comunità", rende ancora più difficile il raggiungimento di una soluzione del problema "carie".

Per affrontare tale problema il Ministero del Lavoro, della Salute e delle Politiche Sociali nel mese di ottobre del 2008 ha emanato le "Linee guida nazionali per la promozione della salute orale e la prevenzione delle patologie orali in età evolutiva" (30) al fine di poter dare dei suggerimenti ai diversi professionisti (Pediatri, Odontoiatri, Microbiologi, Igienisti Dentali, ecc.) in modo che questi interagiscano per il mantenimento ed il ripristino della salute orale. È stata la prima volta che il Ministero della Salute si è occupato del problema inserendo la figura del microbiologo in campo odontoiatrico. La patologia cariosa è una malattia infettiva a carattere cronico-degenerativo, trasmissibile, ad eziologia multifattoriale, che interessa i tessuti duri dentali e che porta alla distruzione degli stessi (8).

Nel cavo orale sono presenti centinaia di specie batteriche di cui la maggior parte saprofitiche. Almeno 20 specie batteriche hanno la capacità di produrre acidi deboli (specie cariogene), il batterio più importante nell'eziopatogenesi della carie è $S$. mutans, anche se nelle lesioni cariose si possono rinvenire altri batteri cariogeni, (altri streptococchi: S.oralis, S.sanguis, S.mitis), lattobacilli e attinomiceti.

Le quattro proprietà principali di queste specie batteriche sono: adesività, fermentazione di carboidrati (soprattutto saccarosio, ma anche glucosio, fruttosio e lattosio) assunti con la dieta, sintesi di polisaccaridi intra ed extracellulari e crescita in ambiente acido.

Gli zuccheri ingeriti con la dieta (intesi come carboidrati fermentabili) svolgono un ruolo fondamentale nei meccanismi eziopatogenetici della carie. Tale evidenza si basa su ricerche svolte sia in vivo sia in vitro $(32,26,16,10,38,24,15)$.

La frequente assunzione di carboidrati fermentabili durante l'arco della giornata produce ripetuti abbassamenti del valore del $\mathrm{pH}$ orale, che favoriscono la demineralizzazione dei tessuti duri dentari, dando il via alla formazione della lesione cariosa. È, pertanto, auspicabile che la frequenza di assunzione di questi principi nutritivi sia ridotta e relegata ai pasti principali, al termine dei quali le manovre di igiene orale possano allontanarli rapidamente dalla bocca.

La relazione tra consumo di "zuccheri" e malattia cariosa nei paesi industrializzati per lungo tempo è stata ritenuta virtualmente lineare: più una popolazione consumava "zuccheri", più elevata era la prevalenza della patologia cariosa. Recentemente la relazione sembra assumere una tendenza non più lineare per il ruolo protettivo del fluoro, che è in grado di ridurre il rischio di carie, anche in presenza di elevati e crescenti consumi di zuccheri.

L'assunzione di zuccheri aggiunti può arrivare a $15 \mathrm{~kg} /$ pro capite/anno $(45 \mathrm{~g} / \mathrm{die})$ in presenza di un adeguato apporto di fluoro (23).

Nella prevenzione di tale patologia è importante la collaborazione del microbiologo clinico con l'odontoiatra e l'igienista dentale per evidenziare eventuali cariche batteriche critiche mediante il test microbiologico salivare. Tale studio è iniziato nel mese di giugno 2009 e durerà un anno con l'obbiettivo di avere, nel 2010, il 90\% di bambini tra i 5 e 6 anni carie free e a 18 anni nessun dente perso per carie.

\section{MATERIALI E METODI}

Sono stati reclutati 164 pazienti suddivisi in tre gruppi di età: 124 adulti di età compresa tra 20 e 40 anni, 40 bambini di cui 21 di 5 anni di età e 19 di 12 anni di età.

\section{Corresponding author: Marcello Gatti}

Università degli Studi di Bologna, Dipartimento di Scienze Odontostomatologiche, sezione di Microbiologia

Via San Vitale, 59 - 40125 Bologna, Italia - Tel: +39 05। 2088I56 - Fax:05। 225208

E mail:marcello.gatti@unibo.it 
Dallo studio sono stati eliminati pazienti in terapia antibiotica, e in terapia con collutori. L'analisi microbiologica era finalizzata alla ricerca di C.F.U./ml di saliva di S. mutans e Lactobacillus spp.. Clinicamente veniva eseguito un esame intraorale per valutare l'indice DMFT negli adulti (Denti Cariati, Mancanti o Otturati) calcolato su 28 denti permanenti, e l'indice dmft nei bambini (denti cariati, mancanti o otturati) calcolato su 20 denti decidui, utilizzando la scheda dell' WHO. Procedura dell'esame clinico:

a) paziente preferibilmente a digiuno;

b) attento esame intraorale;

c) raccolta di dati anamnestici mediante compilazione di una scheda comprensiva anche del consenso informato per l'utilizzo dei dati personali.

Il protocollo microbiologico prevedeva:

a) raccolta di saliva in un contenitore sterile monouso dopo masticazione di pastiglia di paraffina per 5 minuti;

b) preparazione di un vetrino a fresco ed osservazione microscopica sia in campo oscuro che normale;

c) esame colturale, utilizzando lo slide del commercio CRT bacteria (Ivoclar) incubato a $37^{\circ} \mathrm{C}$ per 48 ore;

d) determinazione del $\mathrm{pH}$ salivare mediante cartina di tornasole;

e) dalle colonie cresciute venivano allestiti vetrini colorati con Gram;

f) le colonie sospette (streptococchi) venivano isolate su piastre di agar sangue di cavallo (Biolife) e MRS agar (lattobacilli) (Biolife) ed incubate a $37^{\circ} \mathrm{C}$ per 48 ore in aerobiosi per gli streptococchi ed anaerobiosi per i lattobacilli utilizzando il sistema GENbag (bioMérieux) fino ad ottenere colture pure;

g) l'identificazione di specie avveniva utilizzando i sistemi del commercio API (bioMérieux).

\section{RISULTATI}

Dall'osservazione microscopica di vetrini a fresco si sono evidenziati cellule di sfaldamento della mucosa orale infarcite di bacilli mobili, immobili, e catenelle di cocchi; l'osservazione degli stessi, in campo oscuro, ha rilevato la presenza di spirochete che ci hanno fatto pensare alla presenza di batteri proteolitici nelle cavità cariose attive.

Dalle osservazione dei preparati microscopici di tutti i campioni abbiamo ottenuto informazioni importanti per quanto riguarda la presenza dei batteri correlabili al tipo di dieta del paziente e al valore del $\mathrm{pH}$ salivare e alle abitudini quotidiane di igiene orale. Per quanto riguarda la ricerca di S.mutans, su 164 campioni: 55 (44 adulti e 11 bambini), sono risultati con una carica $\leq 10^{3}$ C.F.U. $/ \mathrm{ml}, 50$ (43 adulti e 7 bambini), sono risultati con una carica compresa tra $>10^{3}$ e $<10^{5}$ C.F.U./ml, e 59 (37 adulti e 22 bambini) sono risultati con una carica $\geq 10^{5}$ C.F.U./ml, come riportato nella Figura I.

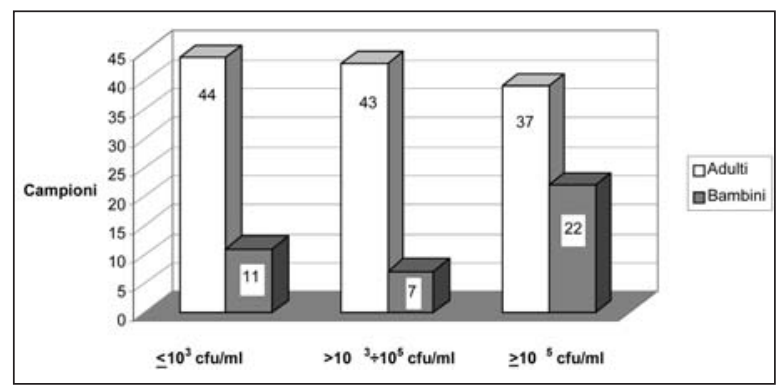

Figura I. Distribuzione della carica di S.mutans nei 164 campioni di saliva studiati.

Per i lattobacilli i risultati sono stati: su 164 campioni: 60 (49 adulti e 11 bambini) sono risultati con una carica $\leq 10^{3}$ C.F.U./ml, 52 (45 adulti e 7 bambini) sono risultati con una carica compresa tra $>10^{3}$ e $<10^{5}$ C.F.U./ml e 52 (30 adulti e 22 bambini) sono risultati con una carica $\geq 10^{5}$ C.F.U. $/ \mathrm{ml}$, come riportato nella Figura II.

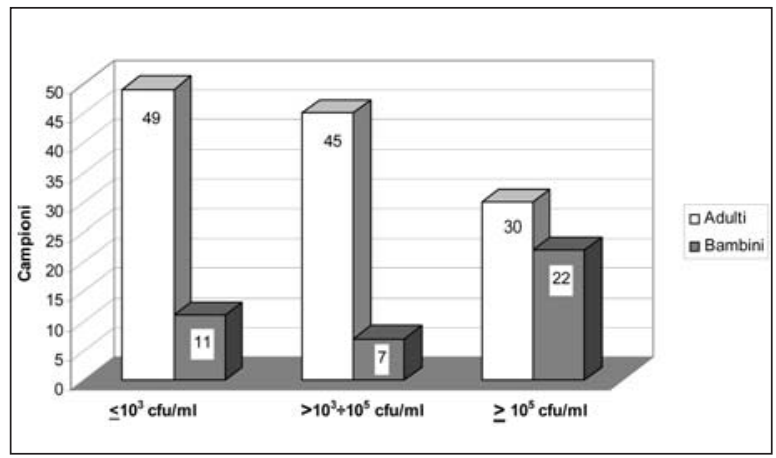

Figura II. Distribuzione della carica di Lactobacillus spp. nei 164 campioni di saliva studiati.

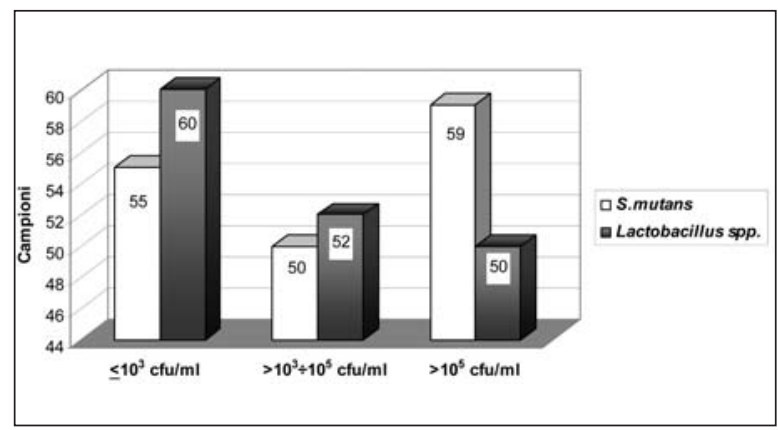

Figura III. Viene riportata la presenza (distribuzione della carica batterica) di S.mutans e Lactobacillus spp. nei 164 campioni di saliva studiati.

Altro dato importante è stato quello del $\mathrm{pH}$. Tutti i pazienti con $\mathrm{pH}$ compreso tra 6.5 e 6.0 sono risultati negativi per la ricerca di S.mutans e Lactobacillus spp.

I pazienti con $\mathrm{pH}$ compreso tra 6,0 e 5,5 sono risultati con una carica compresa tra $>10^{3}$ e $<$ di $10^{5} \mathrm{cfu} / \mathrm{ml}$ di saliva. Mentre i campioni con $\mathrm{pH}<5,5$ sono risultati positivi all'indagine microbiologica con cariche $\geq 10^{5} \mathrm{cfu} / \mathrm{ml}$ di saliva per S.mutans e Lactobacillus spp. Alcuni di questi campioni erano risultati positivi microscopicamente anche per le spirochete. I risultati dell'indice DMFT/dmft sono riportati nella Figura seguente.

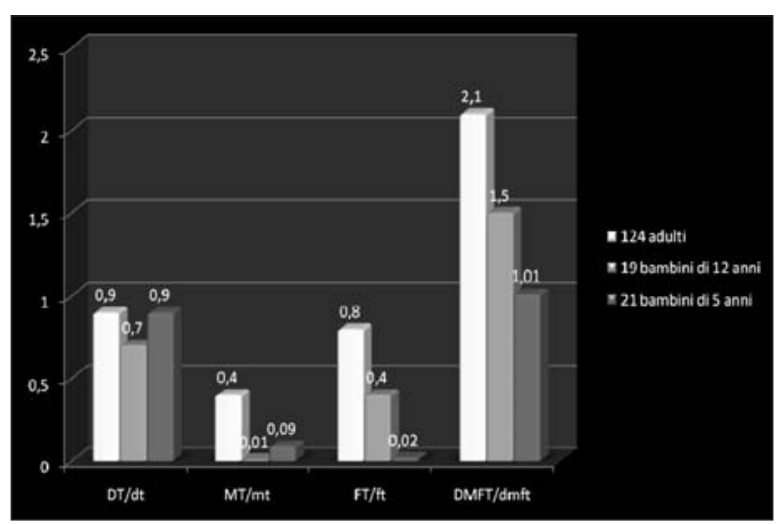

Figura IV. Vengono riportati i risultati dell'indice DMFT calcolato nei I 43 soggetti studiati di cui 124 adulti e 19 bambini di 12 anni; e $i$ risultati dell'indice dmft calcolato nel gruppo di studio formato da 21 bambini di 5 anni.

Confrontando i risultati, molto simili, ottenuti con il test microbiologico e l'indice clinico DMFT/dmft abbiamo calcolato la percentuale dei soggetti: affetti da carie, a rischio di carie e carie indenni, come indicato nella Figura seguente. 


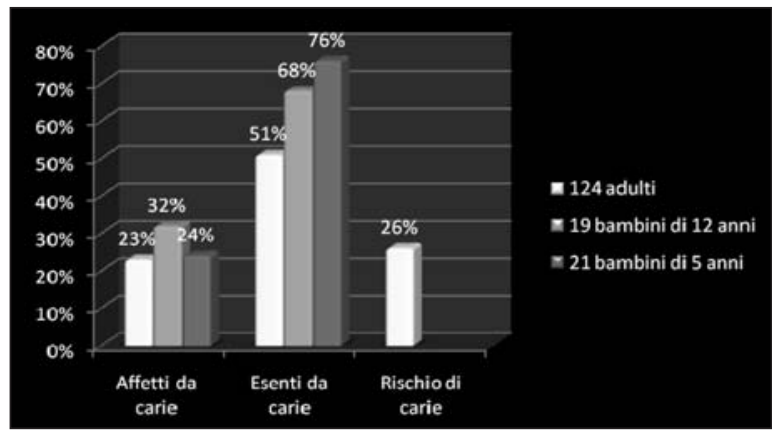

Figura V. Riporta la percentuale di adulti con lesioni cariose, senza lesioni cariose, a rischio di carie e la percentuale di bambini di 12 e di 5 anni affetti ed esenti da carie.

\section{DISCUSSIONE E CONCLUSIONI}

I risultati del test salivare e dell'indice DMFT/dmft indicano che i bambini di 5 anni carie free sono risultati il $76 \%$, mentre i bambini di 12 anni sono risultati il $68 \%$, in quanto i bambini con test salivare risultato con cariche intermedie sono stati considerati ad alto rischio principalmente per due motivi: a) il tipo di alimentazione ricca in carboidrati (largo consumo di merendine) e b) scarsa igiene orale (una sola volta al giorno). Gli adulti carie free sono risultati il $51 \%$ e il $26 \%$ a rischio di carie per la presenza sia di una carica batterica critica (compresa tra $10^{4}$ e $10^{5}$ ), sia di precedenti lesioni cariose.

Si sottolinea che a livello pediatrico rimane elevata, la percentuale di bambini con carie anche se migliorata negli ultimi anni. Per raggiungere l'obbiettivo che si è prefissato il Ministero del Lavoro, della Salute e delle Politiche Sociali nel 2010 si dovranno adottare le raccomandazioni riportate nelle linee guida: Raccomandazione 1: vista la considerevole prevalenza della patologia cariosa nella popolazione infantile italiana, si suggerisce di considerarla, dal punto di vista teorico e nel suo complesso, tutta a rischio di carie.

Raccomandazione 2: la somministrazione di fluoro per via sistemica (gocce o compresse 0.3-0.6 ppm F) andrebbe sempre valutata per tutti i bambini dai 6 mesi ai 6 anni. La costante presenza di adeguate concentrazioni di fluoro nel cavo orale riduce significativamente il rischio di ammalare di carie $(17,18,19,33,35,7,20,25,26,34,14,37,1,11)$.

Raccomandazione 3: la somministrazione di fluoro per via topica (dentifricio con $500 \mathrm{ppm}$ F) andrebbe raccomandata da "appena possibile" fino ai 6 anni, 2 volte al giorno; dopo i 6 anni la concentrazione deve passare a 1.000 ppm F. Per i pazienti con un rischio di carie particolarmente elevato sono necessarie misure preventive addizionali, come ulteriori prodotti a base di fluoro $(2,9,13,20,21,25,26)$. Sarà compito dell'odontoiatra infantile prescrivere, valutando caso per caso, il mezzo di somministrazione più idoneo e la concentrazione di fluoro da utilizzare, dopo aver valutato il rischio microbiologico con tecniche adeguate.

Raccomandazione 4: le sigillature sono indicate per i bambini ma anche per tutti i soggetti.

Raccomandazione 5: l'uso del succhiotto e/o del biberon contenente bevande zuccherate deve essere fortemente sconsigliato, soprattutto durante le ore notturne.

Raccomandazione 6: l'assunzione dei carboidrati fermentabili è da evitare durante la giornata e va relegata ai pasti principali. Queste raccomandazioni derivano dalle conoscenze e competenze condivise dalle diverse professionalità sanitarie (neonatologi, pediatri, odontoiatri infantili, igienisti dentali, microbiologi, farmacologi, ecc).

L'Igienista dentale deve insegnare ai genitori le tecniche per mantenere una corretta igiene orale nel bambino fino dalla tenera età: uso di garzina umida e spazzolini morbidi differenziati a seconda dell'età.

Il compito del microbiologo clinico è quello di affiancare le altre professionalità sanitarie nel valutare la quantità e quali- tà dei batteri cariogeni nelle lesioni attive da un campione di saliva durante i controlli per individuare precocemente cariche batteriche critiche.

Da questi dati preliminari abbiamo visto, insieme all'Odontoiatra, che esiste una buona correlazione tra il test microbiologico e l'indice clinico DMFT/dmft, pertanto si consigliano almeno 2 visite annuali di controllo con l'esecuzione del test salivare, la continua motivazione nel praticare correttamente l'igiene orale quotidiana e l'assunzione del fluoro. Così facendo si spera di raggiungere l'obiettivo prefissato.

\section{BIBLIOGRAFIA}

1. Adair SM. Evidence-based use of fluoride in contemporary pediatric dental practice. Pediatr Dent. 2006;28(2):133-42.

2. Axelsson S, Soder B, Nordenram G, et al. Effect of combined caries-preventive methods: a systematic review of controlled clinical trials. Acta Odontol Scand. 2004;62(3):163-9.

3. Campus G, Cagetti MG, Solinas G, et al. The Italian Study Group on Children Oral Health. ECC (Early Childhood Caries) in Italy, pattern and severity. Caries Res 2007;41:299-300.

4. Campus G, Lumbau A, Sanna AM, et al. Oral health condition in an Italian preschool population. Eur J Paediatr Dent. 2004; 5: 86-91.

5. Campus G, Sacco G, Cagetti MG, Abati S. Changing trend of caries from 1989 to 2004 among 12-year old Sardinian children. BMC Public Health 2005, 7:28 doi:10.1186/1471-2458-7-28.

6. Cleaton-Jones P, Fatti P, Bonecker M.Dental caries trends in 5- to 6-yearold and 11- to 13-year-old children in three UNICEF designated regions Sub Saharan Africa, Middle East and North Africa, Latin America and Caribbean: 1970-2004. Int Dent J. 2006;56:294-300.

7. Douglass JM, Douglass AB, Silk HJ. A practical guide to infant oral health. Am Fam Physician. 2004;70:2113-20.

8. Featherstone JD. The caries balance: contributing factors and early detection. J Calif Dent Assoc. 2003; 31: 129-33.

9. Harris R, Nicoll AD, Adair PM, Pine CM. Risk factors for dental caries in young children: a systematic review of the literature. Community Dent Health. 2004; 21(1 Suppl): 71-85.

10. Harris R, Nicoll AD, Adair PM, Pine CM. Risk factors for dental caries in young children: a systematic review of the literature. Community Dent Health. 2004;21(1 Suppl):71-85.

11. Hiiri A, Ahovuo-Saloranta A, Nordblad A, Makela M. Pit and fissure sealants versus fluoride varnishes for preventing dental decay in children and adolescents. Cochrane Database Syst Rev. 2006;(4):CD003067.

12. Hugoson A, Koch G, Gothberg C, Helkimo AN, Lundin SA, Norderyd O, Sjodin B, Sondell K. Oral health of individuals aged 3-80 years in Jonkoping, Sweden during 30 years (1973-2003). II. Review of clinical and radiographic findings. Swed Dent J. 2005;29:139-55.

13. Johnson MF. The role of risk factors in the identification of appropriate subjects for caries clinical trials: design considerations. J Dent Res. 2004;83.

14. Jones S, Burt BA, Petersen PE, Lennon MA. The effective use of fluorides in public health. Bull World Health Organ. 2005;83:670-6.

15. Karjalainen S. Eating patterns, diet and dental caries. Dent Update. 2007; 34: $295-8,300$.

16. Krol DM. Dental caries, oral health, and pediatricians. Curr Probl Pediatr Adolesc Health Care. 2003; 33: 253-70.

17. Leroy R, Bogaerts K, Lesaffre E, Declerck D. The effect of fluorides and caries in primary teeth on permanent tooth emergence. Community Dent Oral Epidemiol. 2003;31:463-70.

18. Levy SM. An update on fluorides and fluorosis. J Can Dent Assoc. 2003;69:286-91.

19. Marinho VC, Higgins JP, Logan S, Sheiham A. Topical fluoride (toothpastes, mouthrinses, gels or varnishes) for preventing dental caries in children and adolescents. Cochrane Database Syst Rev. 2003;(4):CD002782.

20. Marinho VC, Higgins JP, Sheiham A, Logan S. Combinations of topical fluoride (toothpastes, mouthrinses, gels, varnishes) versus single topical fluoride for preventing dental caries in children and adolescents. Cochrane Database Syst Rev. 2004a;(1):CD002781.

21. Marinho VC, Higgins JP, Sheiham A, Logan S. One topical fluoride (toothpastes, or mouthrinses, or gels, or varnishes) versus another for preventing dental caries in children and adolescents. Cochrane Database Syst Rev. 2004b;(1):CD002780.

22. Marthaler TM. Changes in dental caries 1953-2003. Caries Res. 2004;38:173-81

23. Moynihan P, Petersen PE. The scientific basis for diet, nutrition and prevention of dental diseases. Public Health Nutrition 2002; 7: 201-226.

24. Nunn JH. The burden of oral ill health for children. Arch Dis Child. 2006; 91: 251-3.

25. Petersson GH. Assessing caries risk-using the Cariogram model. Swed Dent J Suppl. 2003; 158:1-65

26. Petersson LG, Twetman S, Dahlgren $\mathrm{H}$, et al. Professional fluoride varnish treatment for caries control: a systematic review of clinical trials. Acta Odontol Scand. 2004;62:170-6.

27. Selwitz RH, Ismail AI, Pitts NB. Dental caries. Lancet. 2007; 369 (9555): 51-9

28. Senna A, Campus G, Gagliani M, Strohmenger L. Social economical influence on caries experience and CPITN values among a group of Italian callup soldiers and cadets. Oral Health and Preventive Dentistry 2005;1:39-46.

29. Strohmenger L, Campus G, Castiglia P, et al. Indagine epidemiologica 
nazionale sulle condizioni dento-parodontali dei bambini di 4 e 12 anni. Doctor Os. 2006; 17: 853-866.

30. Strohmenger L, Cagetti M.G. Campus G, Rosmarini M., De Carli A., Gatti M., Levrini L., Polimeni A., Basso T., Regazzi M., Maida A., Callioni R. Nardone M., Federici A., Natale N., Montagna M.T., Castiglia P., Fabris C., Rondini G., Majorana A., Di Pietro P., Longhi R., Saggese G., Boldi M., Mele G., Munizzi M.R. Linee guida nazionali per la promozione della salute orale e la prevenzione delle patologie orali in età evolutiva, 2008. http://www.ministerosalute.it/

31. Tinanoff N, Kanellis MJ, Vargas CM. Current understanding of the epidemiology mechanisms, and prevention of dental caries in preschool children. Pediatr Dent. 2002; 24:543-51.

32. Touger-Decker R, van Loveren C. Sugars and dental caries. Am J Clin Nutr. 2003; 78: 881S-892S
33. Twetman S, Axelsson S, Dahlgren H, et al. Caries-preventive effect of fluoride toothpaste: a systematic review. Acta Odontol Scand. 2003;61:347-55.

34. Twetman S, Petersson L, Axelsson S, et al. Caries-preventive effect of sodium fluoride mouthrinses: a systematic review of controlled clinical trials. Acta Odontol Scand. 2004; 62:223-30.

35. Weintraub JA. Fluoride varnish for caries prevention: comparisons with other preventive agents and recommendations for a community-based protocol. Spec Care Dentist. 2003; 23(5):180-6.

36. Whelton $\mathrm{H}$. Overview of the impact of changing global patterns of dental caries experience on caries clinical trials. J Dent Res. 2004;83 Spec No $\mathrm{C}: \mathrm{C} 29-34$.

37. Yeung CA, Hitchings JL, Macfarlane TV, et al. Fuoridated milk for preventing dental caries. Cochrane Database Syst Rev. 2005;(3):CD003876. 38. Zero DT. Sugars - the arch criminal? Caries Res. 2004; 38: 277-85. 